\title{
Texture Analysis for Off-Line Signature Verification
}

\author{
J.F. Vargas ${ }^{1,2}$ and M.A. Ferrer ${ }^{1}$ \\ ${ }^{1}$ Universidad de Las Palmas de Gran Canaria - IDeTIC, \\ ${ }^{2}$ Universidad de Antioquia - GEPAR \\ ${ }^{1}$ Spain, \\ ${ }^{2}$ Colombia
}

\section{Introduction}

It has been suggested that verification of identity is a crucial part of the current information society. The number of situations where it is necessary a quick and inexpensive document authentication for access or information sharing, and even more for electronic commerce grows daily.

In the case of personal Verification, it is possible consider two types of biometric means: Physiological, which are derived from direct measurements of human body parts, and Behavioral, which are derived from measurements taken from an action performed by an individual to be described in an indirect manner. As examples of the former can include fingerprint, face, palm, and retina among others. In the second group may find the voice, signature, and the rhythm of typing on a computerLiu \& Silverman (2001).

The handwritten signature is still one of the most commonly used and widely accepted ways for the authentication of the identity of a person. Every day thousands of documents are signed by someone in order to authorize a banking transaction, access to information or a building, a legal representation or just a contract. Despite the technology available, the vast majority of verification processes for these signatures are performed manually by human beens through visual inspection. Consequently, there is great interest in the development of automatic signature verification systems which are effective and have the ability to make quick and accurate verification of the handwritten signature of an individual. This implies that the verification of handwritten signatures (VFM) is not just a problem of pattern recognition theoretically interesting, but a real world problem with very significant commercial implications.

Handwritten signature verification is a very complex problem and scientifically attractive. Generally there are few samples to train the classification model, and also has a large intraclass variability. This represents a challenge to the scientific community. Given the importance from an economic point of view represented by this task, there is a great dependence on the effectiveness of security systems intended to prevent fraudulent access to information systems.

Currently the number of documents containing a signature as a means of identifying the person who signs is enormous, for example, in the United States extend around 17 billion 
checks a year TowerGroup (Online). The verification of these documents by expert reviewers would mean astronomical costs in time and money.

Trying to increase the information available for biometric verification systems based on handwritten signatures, without this means the construction or purchase of additional capture devices, implies the possibility of developing more reliable systems with an aggregate value in constant growth. As mentioned above, in the case of off-line signature verification systems, the information available comes from a static image of the signature. This implies the non-availability of dynamic information corresponding to the signature. We can say that it is possible to characterize the information according to three approaches: a global analysis of the shape of the signature, a local analysis, or an analysis that moves away from the shape to focus primarily on the reconstruction of dynamic information.

Although, with enough training, a forger can reproduce with great skill the shape and distribution of the strokes that make up the signature, with respect to the velocity, pressure and the writing order of strokes, a forger will always find features very difficult to reproduce. This suggests that the ability to recreate and/or represent dynamic information from static images of a signature, represents a very attractive challenge for the scientific community. However, considering that the main source of information for this purpose is a grayscale image, it is necessary to develop the procedures necessary to identify the effect of perceived changes in brightness when analyzing images of signatures that have been written on different paper types (color, weight), and using different types of pen (color ink).

Also, despite the off-line systems are still disadvantaged compared to on-line systems in terms of percentages of success, still present the need for verification of identity of a person who is not present in the moment that it performs this task, as in the case of payments of checks, powers of attorney, contract signings, and any other transaction involving documentation that has been signed by someone as proof of their commitment.

This implies the need to continue working on the development of off-line systems more reliable and better suited to actual needs. The emergence of international conferences with specific focus on the analysis of documents, shows the high interest of the scientific community on this issue. Consequently, it has generated a large number of activities aimed at the automatic verification of handwritten signatures with very promising results. However, there are unexploited sources of information. In that sense, this work raises, it seeks to advance the study of the gray levels as a source of information for the characterization of a handwritten signature oriented towards his classification as genuine or fake.

This chapter is organized as follows: Section 2 describes characterization based on gray level information containing signature images. This section includes information about ink analysis and statistical texture analysis. Texture analysis is also described for the transformed domain when Wavelet transform is used. Section 3 presents the procedure proposed here for feature extraction. It describes how block analysis is used for image characterization. Datasets used for experiments are also presented in this section. Section 4 presents the experiments and results obtained here, and finally, the conclusions and remarks.

\section{Characterization based on graylevel information}

A neglected aspect in the off-line signature verification is the use of the information in the gray levels of the signature, although a number of studies have been carried out in search of a off-line signature verification system, results have been few satisfactory for the case of those systems based on information from the gray levels. While this weakness has been offset by the combination of parameters of different nature, the information contained in the gray level image of a signature is still an untapped potential. The main difficulty encountered by 
researchers has been the influence of the type of ink on the distribution of gray levels, this has meant that the developed systems do not perform the verification of a signature, but the classification of the type of ink used for realization.

It is assumed that the signature depends on the neuromotor apparatus of the person whose development is unique and determines his writing, both in the way of writing the strokes as the manner to handle the pen, and the latter is manifested on how is deposited ink on paper. Depending on the type of ink, a masking is performed for graylevel histogram of the image, is then necessary to develop an analysis that derives the information obviating the masking caused by the ink. In this way you can characterize the distribution patterns of each signer enabling the signature verification stage. In that sense, we propose that the signer information is less masked in the relationship between the gray levels of pixels of the strokes of a handwritten signature than in its absolute value.

\subsection{Ink-type analysis}

There are many methods for the reconstruction of dynamic information from the analysis of ink in handwritten samples. In the field of forensic document analysis, these methodologies are based mainly on microscopic inspection of the strokes and on assumptions about the writing process. This has resulted in the development of ink distribution model proposed by Franke Franke \& Grube (1998). This first model, permitted its adaptation to specific properties of the pen used (solid ink, liquid or viscous). Its weakness was that this will be done manually by selecting the type of ink distribution according to the type of pen. In Franke et al. (2002), the authors propose an evolution to the model. It automatically determines the type of pen used, by analyzing the static strokes. This work opens the possibility to develop procedures that minimize the effect of using different types of pen on automatic verification systems.

\subsubsection{Pen types and their properties}

There are several types of pen. From a technical standpoint, the pens can be classified according to the mechanical principle, the type of tip and the ink used Franke et al. (2002). Maybe it is the type of ink used that have the greatest impact on the outcome of a manuscript stroke. Degree of liquidity of the ink significantly determines the final visual appearance of a stroke made on paper. For example, the ink used on a pencil is graphite and therefore we say it is solid. Pen ink is a viscous paste made from resin, glycerine and other additives. The fluid ink of the other writing instruments, is mainly composed of water that is added to the color pigments. Depending on the additives used, change the liquidity of the ink. We note that the ink of a roller-ball pen is more liquid than a Gel-ink pen. Figure 1 shows strokes made with the three types of ink mentioned.

\subsubsection{Forensic analysis of the type of ink}

As mentioned, the ink is composed mainly of water to be mixed with various additives in order to generate the different types of ink that are usually found in the marketplace. Within these additives used are oils, solvents and resins, whose composition affects the characteristics of flow and drying of the ink. Additionally, other substances such as dryers, plasticizers, waxes, fats, soaps and detergents are also used to finely alter the characteristics of the ink. The large number of elements used in different types of ink, and possible contamination of the writing surface, become a complex problem for forensic specialists. The goal of most of the analysis is to determine if two pieces of written text have been made with the same ink. The techniques used for this purpose can be divided into non-destructive methodologies and destructive. Although it is preferred in non-destructive, the number of such techniques is limited availableThanasoulias et al. (2003). 


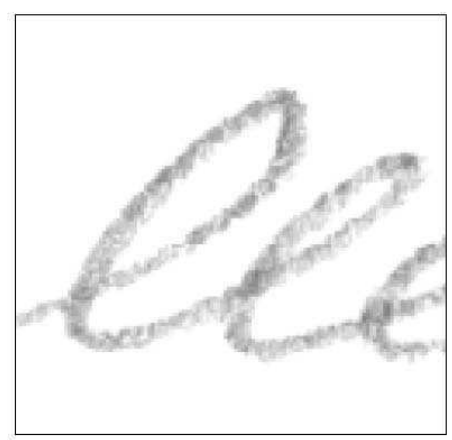

(a) Solid

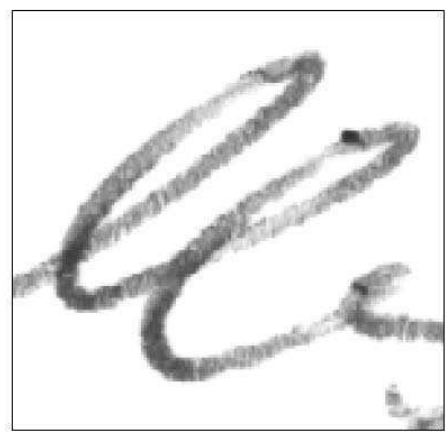

(b) Viscous

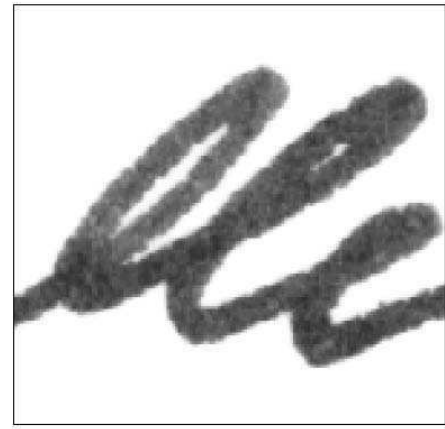

(c) Fluid

Fig. 1. Samples made with different kind ok ink Franke et al. (2002).

Chromatographic separation of the ink in its components has proved to be a highly productive, since it not only allows the comparison of inks but also to do match on a database of chromatograms. Thin layer chromatography (TLC) is widely used for its speed, low cost and minimal destruction of the documents reviewed. The chromatograms can be scanned using a densitometer, but unfortunately the signal to noise ratio is low due to the large size of the area scanned.

\subsubsection{Type of ink analysis using DSP}

Use the techniques of digital image processing in forensic document analysis is relatively new Ellen (1997). The image processing offers significant cost benefits by eliminating or at least minimizes the need for expensive instrumentation and the use of a destructive methodologies. More recent works done by forensic experts show that these specialists have begun using common software packages for the analysis of documents. Most of the techniques used for this purpose are the basic procedures of digital image processing, for example some forms of contrast enhancement Bhagvati \& Haritha (2005). In this sense, there are many benefits to the expert in documents analysis from the image processing community.

Similarly, the forensic field allows us to define and develop a new line of research to be exploited by experts in image processing. An example is the work presented by Bhagvati and others Bhagvati \& Haritha (2005), where it is studied the problem of identifying different 
types of ink techniques using digital image processing. According to the conclusions of the authors, the method presented has a better performance than human perception in the case of printed inks. Used HSV space information, in particular saturation levels, to determine variations in patterns of absorption of the paper according to the ink. Figure 2 shows the estimated models.

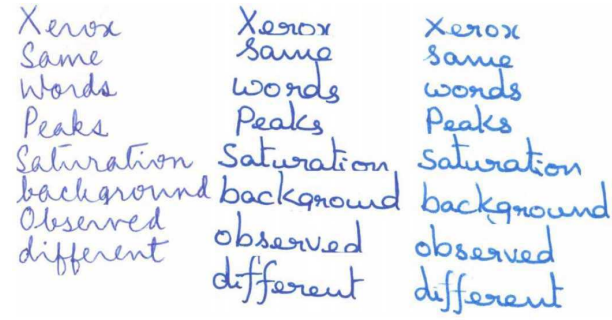

(a) Ball-pen (left), Gel-pen (center), Roller-pen (rigth)

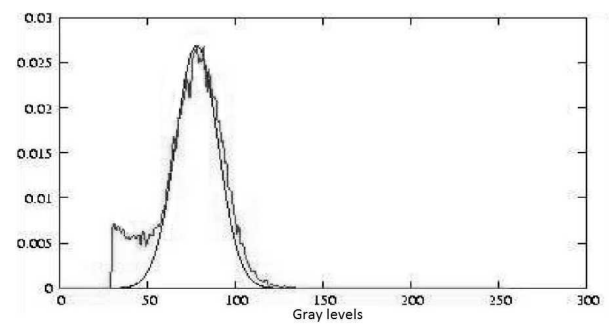

(c) Gel-pen histograms

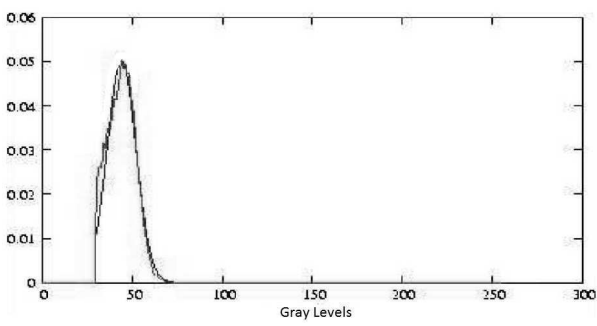

(b) Ball-pen histograms

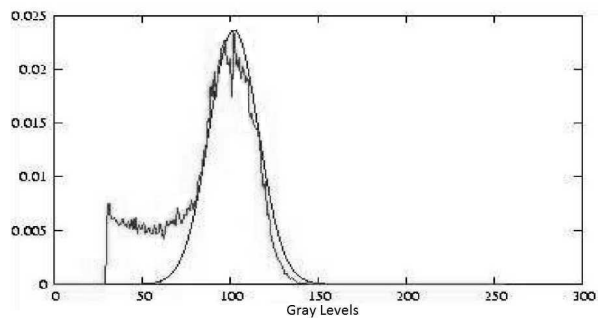

(d) Roller-pen histograms

Fig. 2. Saturation histograms and Gaussian Model estimated for different types of ink, Bhagvati \& Haritha (2005).

For the specific case of handwriting analysis, Frank and others Franke et al. (2002) presented a paper focusing on developing a methodology to automatically determine the type of pen used by the analysis of the strokes in a static image. In that study, there was more attention to the characteristics that describe the visual appearance of the ink distribution along the handwritten lines. Given that the shape of the lines is dependent on each writer and provides little information about the type of ink, this feature was not taken into account by the authors. As shown in Figure 1, texture in images of handwritten strokes is determined by the physical properties of the ink used. Therefore it is suggested that is possible to determine the type of ink used from an analysis of textures in the image. To this end, the authors use a classical approach in the area of texture analysis, the Gray Level Co-occurrence Matrix (GLCM), or array of spatial dependence of gray levels present in an image. To represent each texture, were used second-order statistical features calculated from GLCM, which allows independence from the lighting. Figure 3 presents the GLCM matrices obtained for the three types of ink shown in Figure 1. The results obtained by the authors in the classification of these three types of ink showing an error less than $1 \%$. In another work, Frank and Rose Franke \& Rose (2004) describe their study of the influence of physical and biomechanical processes on the ink strokes in order to provide a solid foundation that allows the improvement of signature analysis systems. Using a robot writer, able to handle different types of writing tools under 


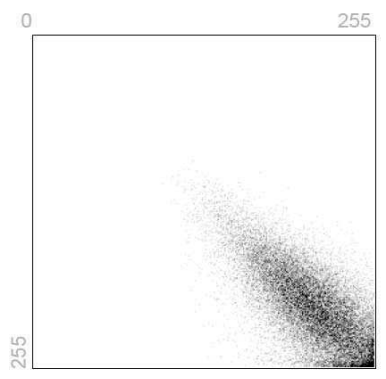

(a) Solid

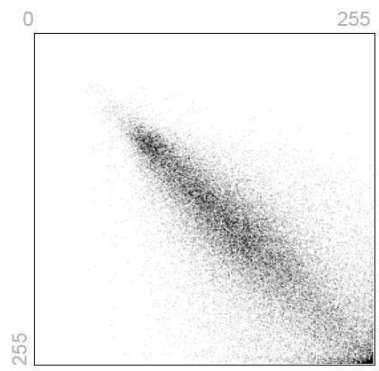

(b) Viscous

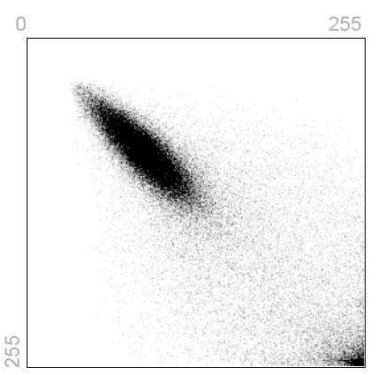

(c) Fluid

Fig. 3. GLCM with 255 graylevels for different type of ink Franke et al. (2002).

controlled conditions, they simulate the motions of writing in order to study the relationship between the characteristics of the writing process and the deposition of ink on paper. As a result of the analysis of these artificial strokes, corresponding to the use of 30 different pens, the authors proposed an Ink Deposition Model (IDM). This model describes analytically the relationship between the force applied to the pen and the relative intensity distribution of three types of ink: Solid, Viscous and fluid.

Figure 4 shows histograms for the three types of ink taken into account in the study, when applying different values of force on the pen. It may see a shift to the left (darker graylevels) when increasing the force exerted on the pen. The authors also mention that changes in the color of the ink only represent a shift of the histogram without changes in the distribution.

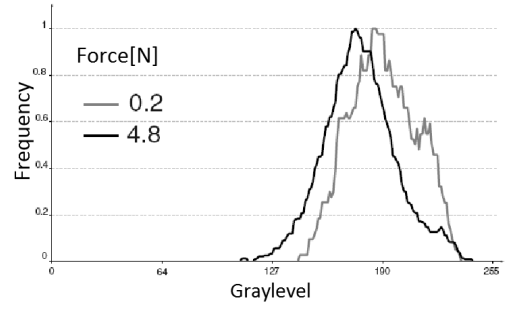

(a) Solid

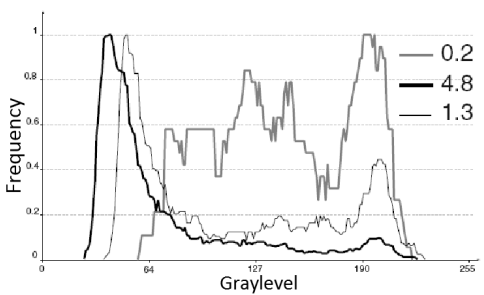

(b) Viscous

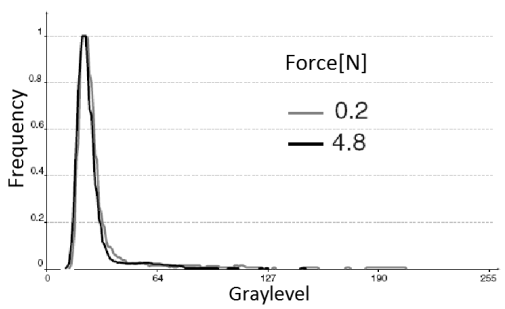

(c) Fluid

Fig. 4. Histograms applying different force values on pen Franke \& Rose (2004). 


\subsection{Statistical texture analysis}

Biometric systems based on signature verification, in conjunction with textural analysis, can reveal information about ink-pixels distribution which reflects personal characteristics from the signer i.e. pen-holding, writing speed and pressure. But we do not think that only ink distribution information is sufficient for signer identification. So, in the specific case of signature strokes, we have also taken into account, for the textural analysis, the pixels in the stroke contour. By this we mean those stroke pixels that are in the signature-background border. These pixels will include statistical information about the signature shape. So this distribution data may be considered as a combination of textural and shape information.

\subsubsection{Grey Level Co-occurrence Matrices}

The Grey Level Co-occurrence Matrix (GLCM) method is a way of extracting second order statistical texture features from the image Conners \& Harlow (1980). This approach has been used in a number of applications, including ink type analysis, e.g. Franke et al. (2002); Haralick (1979); He et al. (1987); Trivedi et al. (1984).

A GLCM of an image $I(x, y)$ is a matrix $P\left(i, j \mid \Delta_{x}, \Delta_{y}\right), 0 \leq \mathrm{i} \leq \mathrm{G}-1,0 \leq \mathrm{j} \leq \mathrm{G}-1$, where the number of rows and columns is equal to the number of grey levels $\mathrm{G}$. The matrix element $\mathrm{P}\left(\mathrm{i}, \mathrm{j} \mid \Delta_{x}, \Delta_{\mathrm{y}}\right.$ is the relative frequency with which two pixels with grey levels $i$ and $j$ occurs separated by a pixel distance $\Delta_{x}, \Delta_{y}$. For simplicity, in the rest of the paper, we will denote the GLCM matrix as $P(i, j)$.

For a statistically reliable estimation of the relative frequency we need a sufficiently large number of occurrences for each event. The reliability of $P(i, j)$ depends on the grey level number $G$ and the $I(x, y)$ image size. In the case of images containing signatures, instead of image size, this depends on the number of the pixels in the signature strokes. If the statistical reliability is not sufficient, we need to reduce $G$ to guarantee a minimum number of pixels transitions per $P(i, j)$ matrix component, despite losing texture description accuracy. The grey level number $G$ can be reduced easily by quantifying the image $I(x, y)$.

The classical feature measures extracted from the GLCM matrix (see Haralick et al. Haralick (1979) and Conners et al. Conners \& Harlow (1980)) are the following:

- Texture homogeneity H:

$$
H=\sum_{i=0}^{G-1} \sum_{j=0}^{G-1}\{P(i, j)\}^{2}
$$

A homogeneous scene will contain only a few grey levels, giving a GLCM with only a few but relatively high values of $P(i, j)$. Thus, the sum of squares will be high.

- Texture contrast C:

$$
C=\sum_{n=0}^{G-1}\left\{n^{2} \cdot \sum_{i=0}^{G-1} \sum_{j=0}^{G-1} P(i, j)\right\},|i-j|=n
$$

This measure of local intensity variation will favour contributions from $P(i, j)$ away from the diagonal, i.e $i \neq j$.

- Texture entropy E:

$$
E=\sum_{i=0}^{G-1} \sum_{j=0}^{G-1} P(i, j) \cdot \log \{P(i, j)\}
$$


Non-homogeneous scenes have low first order entropy, while a homogeneous scene reveals high entropy.

- Texture correlation $\mathrm{O}$ :

$$
O=\sum_{i=0}^{G-1} \sum_{j=0}^{G-1} \frac{i \cdot j \cdot P(i, j)-\left(m_{i} \cdot m_{j}\right)}{\sigma_{i} \cdot \sigma_{j}}
$$

where $m_{i}$ and $\sigma_{i}$ are the mean and standard deviation of $P(i, j)$ rows, and $m_{j}$ and $\sigma_{j}$ the mean and standard deviation of $P(i, j)$ columns respectively. Correlation is a measure of grey level linear dependence between pixels at the specified positions relative to each other.

\subsubsection{Local Binary Patterns}

The Local Binary Pattern (LBP) operator is defined as a grey level invariant texture measure, derived from a general definition of texture in a local neighbourhood, the centre of which is the pixel $(x, y)$. Recent extensions of the LBP operator have shown it to be a really powerful measure of image texture, producing excellent results in many empirical studies. LBP has been applied in biometrics to the specific problem of face recognition Marcel et al. (2007); Nikam \& Agarwal (2008). The LBP operator can be seen as a unifying approach to the traditionally divergent statistical and structural models of texture analysis. Perhaps the most important property of the LBP operator in real-world applications is its invariance to monotonic grey level changes. Equally important is its computational simplicity, which makes it possible to analyse images in challenging real-time settings $\mathrm{T}$. (2003). The local binary pattern operator describes the surroundings of the pixel $(x, y)$ by generating a bit-code from the binary derivatives of a pixel as a complementary measure for local image contrast. The original LBP operator takes the eight neighbouring pixels using the centre grey level value $I(x, y)$ as a threshold. The operator generates a binary code 1 if the neighbour is greater than or equal to the central level, otherwise it generates a binary code 0 . The eight neighbouring binary codes can be represented by an 8-bit number. The LBP operator outputs for all the pixels in the image can be accumulated to form a histogram which represents a measure of the image texture. Figure 5 shows an example of LBP operator.

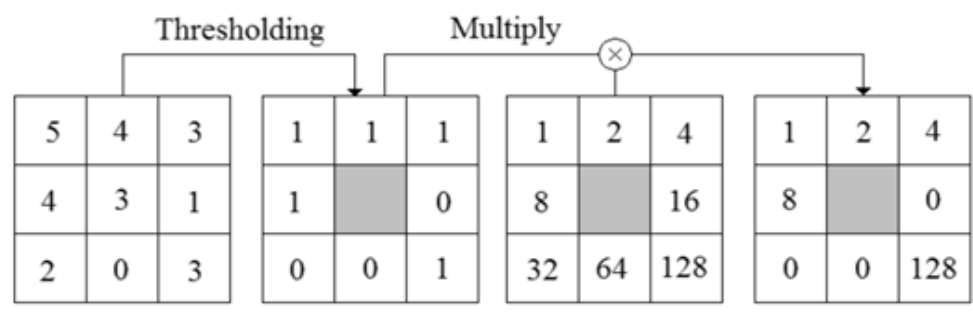

$\mathrm{LBP}=1+2+4+8+128=143$

Fig. 5. Working out the LBP code of pixel $(x, y)$. In this case $I(x, y)=3$, and its LBP code is $\operatorname{LBP}(x, y)=143$.

The above LBP operator is extended in Ojala et al. (2002) to a generalised grey level and rotation invariant operator. The generalised LBP operator is derived on the basis of a circularly symmetric neighbour set of $P$ members on a circle of radius $R$. The parameter 
$P$ controls the quantisation of the angular space and $R$ determines the spatial resolution of the operator. The LBP code of central pixel $(x, y)$ with $P$ neighbours and radius $R$ is defined as:

$$
\operatorname{LBP}_{P, R}(x, y)=\sum_{p=0}^{P-1} s\left(g_{p}-g_{c}\right) \cdot 2^{p}
$$

where $s(l)=\left\{\begin{array}{ll}1 & l \geq 0 \\ 0 & l<0\end{array}\right.$, the unit step function, $g_{c}$ the grey level value of the central pixel: $g_{c}=I(x, y)$ and $g_{p}$ the grey level of the $p^{\text {th }}$ neighbour, defined as:

$$
g_{p}=I\left(x+R \cdot \sin \frac{2 \pi p}{P}, y-R \cdot \cos \frac{2 \pi p}{P}\right)
$$

If the $p^{\text {th }}$ neighbour does not fall exactly in the pixel position, its grey level is estimated by interpolation. An example can be seen in Figure 6
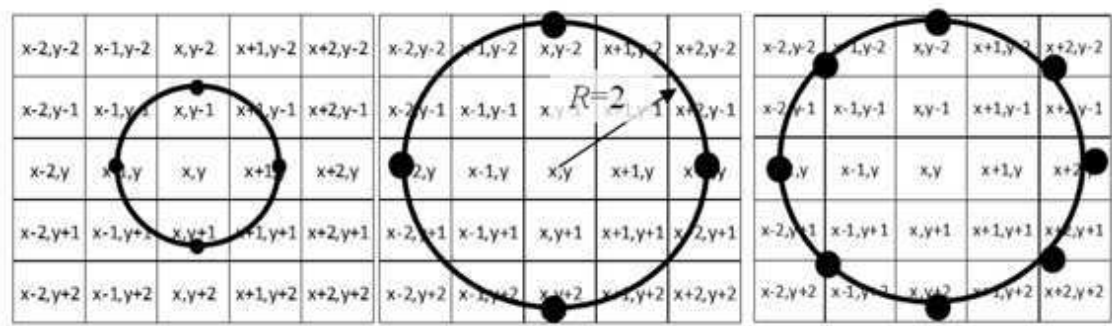

Fig. 6. The surroundings of $I(x, y)$ central pixel are displayed along with the $p^{\text {th }}$ neighbours, marked with black circles, for different $P$ and $R$ values. Left: $P=4, R=1$, the $L P B_{4,1}(x, y)$ code is obtained by comparing $g_{c}=I(x, y)$ with $g_{p=0}=I(x, y-1), g_{p=1}=I(x+1, y)$, $g_{p=2}=I(x, y+1)$ and $g_{p=3}=I(x-1, y)$. Centre: $P=4, R=2$, the $L P B_{4,2}(x, y)$ code is obtained by comparing $g_{c}=I(x, y)$ with $g_{p=0}=I(x, y-2), g_{p=1}=I(x+2, y)$, $g_{p=2}=I(x, y+2)$ and $g_{p=3}=I(x-2, y)$. Right: $P=8, R=2$, the $L P B_{8,2}(x, y)$ code is obtained by comparing $g_{c}=I(x, y)$ with $g_{p=0}=I(x, y-2), g_{p=1}=I(x+\sqrt{2}, y-\sqrt{2})$, $g_{p=2}=I(x+2, y), g_{p=3}=I(x+\sqrt{2}, y+\sqrt{2}), g_{p=4}=I(x, y+2), g_{p=5}=I(x-\sqrt{2}, y+\sqrt{2})$, $g_{p=6}=I(x-2, y)$ and $g_{p=7}=I(x-\sqrt{2}, y-\sqrt{2})$.

In a further step, Ojala et al. (2002) defines a $L B P_{P, R}$ operator invariant to rotation as follows:

$$
\operatorname{LBP}_{P, R}^{r i u 2}(x, y)=\left\{\begin{array}{cl}
\sum_{p=0}^{P-1} s\left(g_{p}-g_{c}\right) \text { if } & U(x, y) \leq 2 \\
P+1 & \text { otherwise }
\end{array}\right.
$$

where

$$
U(x, y)=\sum_{p=1}^{P}\left|s\left(g_{p}-g_{c}\right)-s\left(g_{p-1}-g_{c}\right)\right|, \text { with } \quad g_{c}=g_{0}
$$

Analysing the above equations, $\mathrm{U}(\mathrm{x}, \mathrm{y})$ can be calculated as follows:

1. Work out the function $f(p)=s\left(g_{p}-g_{c}\right), 0<p<P$ considering $g_{P}=g_{0}$;

2. Obtain its derivate: $f(p)-f(p-1), 1 \leq p \leq P$; 
3. Calculate the absolute value: $|f(p)-f(p-1)|, 1 \leq p \leq P$;

4. Obtain $U(x, y)$ as the integration or sum $\sum_{p=1}^{P}|f(p)-f(p-1)|$

If the grey levels of the pixel $(x, y)$ neighbours are uniform or smooth, as in the case of Figure $7(\mathrm{a}), f(p)$ will be a sequence of ' 0 ' or ' 1 ' with only two transitions. In this case $U(x, y)$ will be zero or two and the $L B P_{P, R}^{r i u 2}$ code is worked out as the sum $\sum_{p=0}^{P-1} f(p)$.

Conversely, if the surrounding grey levels of pixel $(x, y)$ vary quickly, as in the case of figure $7(b), f(p)$ will be a sequence containing several transitions ' 0 ' to ' 1 ' or ' $1{ }^{\prime}$ to ' 0 ' and $U(x, y)$ will be greater than 2 . So, in the noisy case, a constant value equal to $P+1$ is assigned to $L B P_{P, R}^{r i u 2}$ making it more robust to noise than previously defined LBP operators.

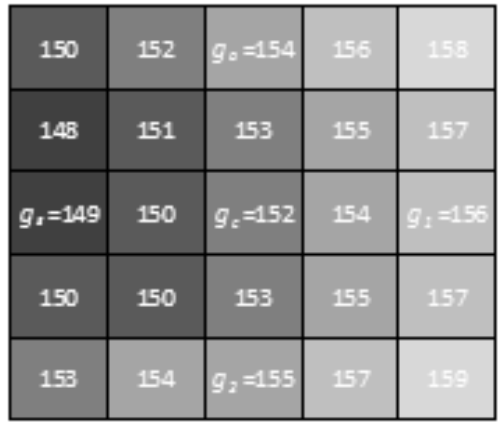

(a) smooth and uniform grey level change

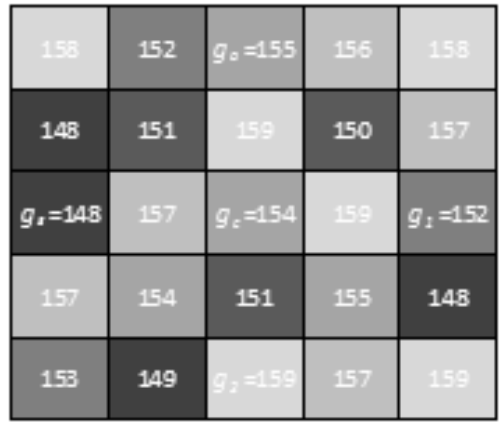

(b) noisy grey level surroundings

Fig. 7. Calculating the $L B P_{P, R}^{r i u 2}$ code for two cases, with $P=4$ and $R=2$ : Left: $g_{c}=152$, $g_{0}, g_{1}, g_{2}, g_{3}=154,156,155,149, f(0), f(1), f(2), f(3), f(4)=1,1,1,0,1$, $U(x, y)=0+0+1+1=2 \leq 2$, therefore $L B P_{P, R}^{r i u 2}(x, y)=1+1+1+0=3$. Right: $g_{c}=154$, $g_{0}, g_{1}, g_{2}, g_{3}=155,152,159,148, f(0), f(1), f(2), f(3), f(4)=1,0,1,0,1$, $U(x, y)=1+1+1+1=4 \leq 2, \operatorname{LBP}_{P, R}^{\text {riu }}(x, y)=P+1=5$.

The rotation invariance property is guaranteed because when summing the $f(p)$ sequence to obtain the $L B P_{P, R}^{r i u 2}$, it is not weighted by $2^{p}$. As $f(p)$ is a sequence of 0 and 1 , $0 \leq \operatorname{LBP}_{P, R}^{\text {riu2 }}(x, y) \leq P+1$. As textural measure, we will use its $P+2$ histogram bins of $\operatorname{LBP}_{P, R}^{\text {riu2 }}(x, y)$ codes.

\subsection{Texture analysis in transformed domain}

We describe the use of Wavelet transform as a complement to features already described and with the aim of achieving a reduction in the variance of the results for different databases due to different type of ink used by the signers.

When performing the Wavelet decomposition of an image 4 matrices obtained: 1 with the coefficients of approximation and other 3 with the detail coefficients. For these 3 last, we make use of 3 wavelet which measure functional changes (changes in intensity or gray levels) in different directions. $\psi^{H}$ measures variations along the columns (eg horizontal edges), $\psi^{V}$ corresponds to variations along the rows (eg vertical edges) and $\psi^{D}$ corresponds to diagonal variations. Each of these wavelet is the product of a unidimensional scale $\varphi$ and 
the corresponding wavelet $\psi$. Excluding those products yielding a one-dimensional result, as $\varphi(x) \psi(x)$, the four remaining products define the scaling function

$$
\varphi(x, y)=\varphi(x) \varphi(y)
$$

and three wavelet with directional sensitivity

$$
\begin{aligned}
& \psi^{H}(x, y)=\psi(x) \varphi(y) \\
& \psi^{V}(x, y)=\varphi(x) \psi(y) \\
& \psi^{D}(x, y)=\psi(x) \psi(y)
\end{aligned}
$$

wavelet Haar was used.

Generally, for image compression tasks, most of the relevant information is contained in the matrix of approximation coefficients. Here we propose that the features based on texture analysis, described above, should be calculated from the matrices of detail. This is based on the fact that the matrix of approximation coefficients mainly contain information corresponding to the type of ink used (low frequencies, little changed from gray level), while the detail coefficients contain information on the personal characteristics of the signer (high frequencies, many changes in levels of gray). Figure 8 shows the histograms of the original image and the matrices resulting from the Wavelet decomposition, and serve as the basis of the hypothesis. This analysis corresponds to a sample made using viscose ink, and whose original histogram is similar to the patterns presented by Franke \& Rose (2004) which were presented in Figures 4.

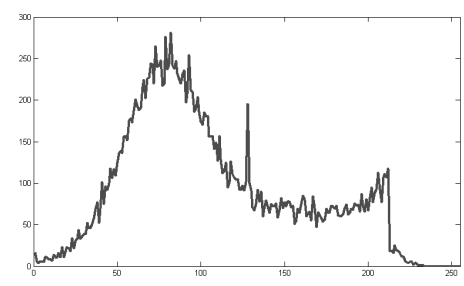

(a)

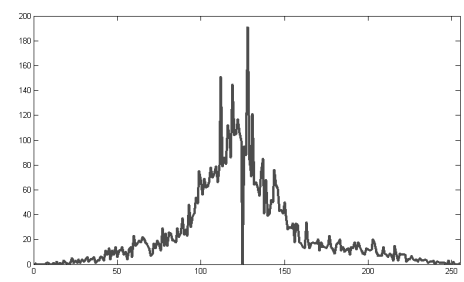

(c)

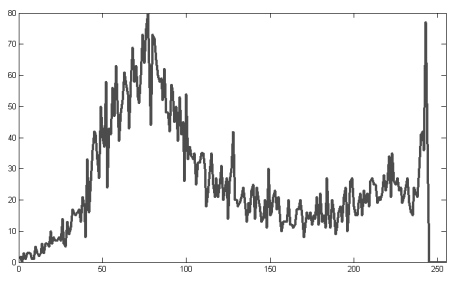

(b)

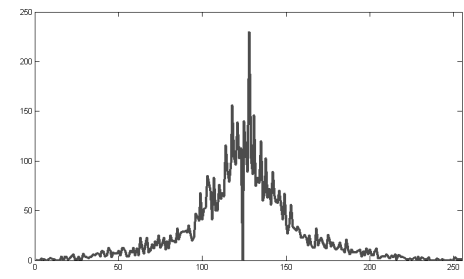

(d)

Fig. 8. Wavelet Decomposition. (a) Original image histogram. (b) Histogram of approximation coefficients. (c) Histogram of vertical detail coefficients. (d) Histogram of horizontal detail coefficients. For sake of visualization, background values has been remove from histograms. 


\section{Feature extraction}

After the wavelet decomposition of the original image, one proceeds with the calculation of textural features. Each one of the three matrix of approximation coefficients is characterized using block analysis. For GLCM, characteristics were calculated: Homogeneity, Contrast, Entropy, Energy and Correlation, and a combination of them was used for verification (CHEEC). Have been used 8 graylevels for GLCM calculation and Offsets vector $\left(\Delta_{x} \Delta_{y}\right)$ with values [0 1, -1 1, -1 0, -1 -1] was used. For LBP, a combination of two LBP operators ( $R=\{1,2\}$ y $P=\{8,16\})$ was used. These two settings allow us to analyze the image at different resolutions.

Finally, the three vectors are concatenated to form the final vector of signature features. Figure 9 shows the procedure to construct the final features vector using the vector of characteristics of the three matrices of approximation coefficients.

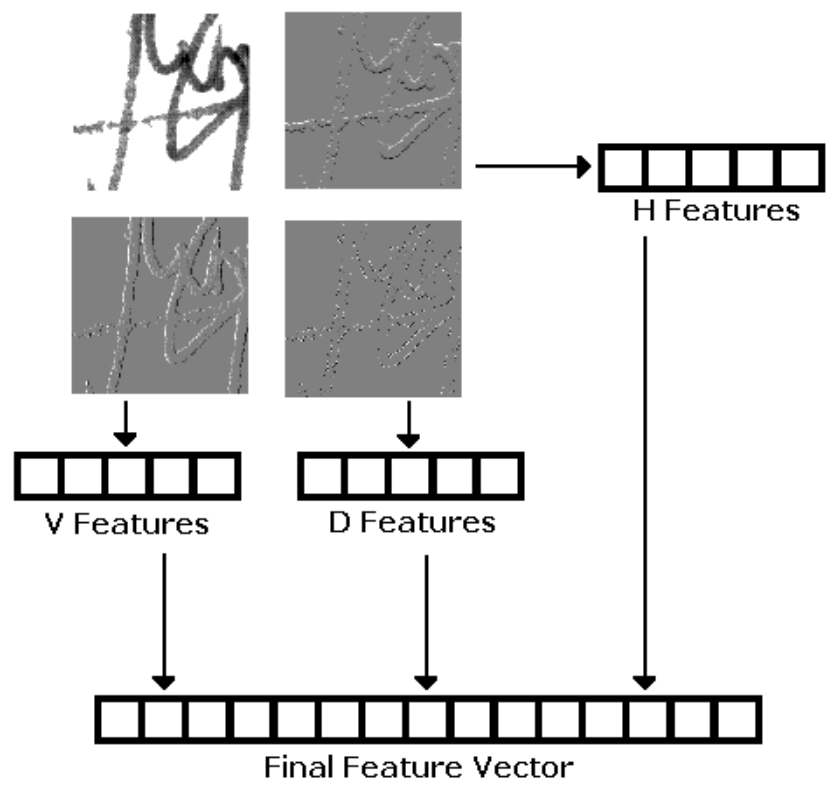

Fig. 9. Feature vector construction.

\subsection{Datasets}

\subsubsection{GPDS corpus}

Digital Signal Processing Group (GPDS) of the Universidad de Las Palmas de Gran Canaria, has devoted efforts to create a large scale database called GPDS-850 Corpus Vargas et al. (2007). This database contains samples of 850 signers with 24 genuine samples and 24 forgeries for each. Signers used their own pen so the dataset contains samples made with different type of ink. Images have a resolution of 600dpi. This dataset was constructed on two stages. In that way, the corpus was divided in two subcorpuses (GPDS100 and GPDS750) for the experiments carried out on this work. 


\subsubsection{MCYT corpus}

With the encouragement of having a reference database, this work takes into account a subcorpus of the database created by the Multimodal Biometric Group - ATVS of the Autonomous University of Madrid. It is available in http://atvs.ii.uam.es/databases.jsp.

The corpus MCYT-SignatureOff-75 Fierrez-Aguilar et al. (2004), contains samples of 75 signers. It has 15 genuine samples and 15 forgeries for each signer. The images have a resolution of 600dpi. It is noteworthy that all the signatures were made with the same type of pen. This database has been used in different works Fierrez-Aguilar et al. (2004), Ferrer et al. (2006), Freire et al. (2007), Alonso-Fernandez et al. (2007), Güler \& Meghdadi (2008), Gilperez et al. (2008), Prakash \& Guru (2009), which allows us to suggest some kind of comparison with the results obtained in this study.

\section{Experiments and results}

Once preprocessed, each sample is characterized and represented by a vector of parameters that feeds the verification stage. In this work, each signer is modeled using Least Squares-Support Vector Machines (LS-SVM).

For this work, we used only samples of the first 75 signers of each database. This in order to make a fair comparison of the results obtained with the 3 corpuses. Model training is performed with 5 and 10 genuine samples as positive samples. This was done in order to carry out an analysis of system performance respect to the number of original samples used for model building. These genuine samples are chosen randomly. Random forgeries were used as negative samples (genuine samples of other signers). The use of these samples was proposed in Bertolini et al. (2009), and for this work it was taken a genuine sample of each of the other signers of the database, ie 74 . Given the small number of samples available for training was used the Leaving-one-out cross-validation procedure (LOOCV), to determine the value of the parameters $(\gamma, C)$ for LS-SVM classifier with RBF kernel.

For the test were used random forgeries and skilled forgeries. In the case of random forgeries, it took a genuine signature of each of the other users of the database (ensuring to be different than the sample used in training). In the case of skilled forgeries, we used all the available samples in the database, ie, 15 in the MCYT and 24 in the GPDS corpuses. To obtain more reliable results, the training and testing procedures were repeated 10 times with different sets of training and test data. As threshold for determining the values of FAR and FRR, and taking into account that the LS-SVM classifier was trained with samples labeled as ' +1 ' for genuine samples and ' -1 ' for forgeries, the value was set at zero for all signatories. That is, if the LS-SVM provides a value greater than zero at its output, the signature is accepted as genuine. If the LS-SVM gives a value less than zero as output, the signature is considered as a forgery and therefore is rejected.

\subsection{Results}

Tables 1 y 2 show results when GLCM and LBP features are used independently for verification.

Table 3 shows results using a feature level combination of GLCM and LBP features. This combination represents a system performence improvement when compared with tables 1 and 2.

The following, we present the results obtained when using the Wavelet transform as a complement to the combination of features referred above. First Wavelet decomposition is performed, then LBP and GLCM features are calculated, and finally these are concatenated to form the feature vector. 


\begin{tabular}{l|l|r|r|r|r|r}
\hline$\#$ & Dataset & FAR(\%) & FRR(\%) & EER(\%) & $\sigma$-FAR & $\sigma$-FRR \\
\hline 5 & MCYT & 5,99 & 20,57 & 11,82 & 3,25 & 8,89 \\
& GPDS100 & 10,82 & 19,33 & 14,58 & 4,68 & 6,92 \\
& GPDS750 & 16,02 & 20,93 & 18,19 & 6,41 & 7,48 \\
10 & MCYT & 11,41 & 12,85 & 11,76 & 3,91 & 9,21 \\
& GPDS100 & 18,66 & 11,86 & 16,16 & 5,54 & 7,84 \\
& GPDS750 & 26,69 & 12,10 & 21,31 & 7,20 & 7,75 \\
\hline
\end{tabular}

Table 1. Results using GLCM features

\begin{tabular}{l|l|r|r|r|r|r}
\hline$\#$ & Dataset & FAR(\%) & FRR(\%) & EER(\%) & $\sigma$-FAR & $\sigma$-FRR \\
\hline 5 & MCYT & 4,34 & 31,18 & 15,07 & 3,36 & 9,04 \\
& GPDS100 & 5,37 & 27,81 & 15,28 & 3,61 & 9,20 \\
& GPDS750 & 4,74 & 30,60 & 16,17 & 4,34 & 9,92 \\
10 & MCYT & 9,14 & 16,53 & 10,98 & 3,74 & 10,18 \\
& GPDS100 & 10,86 & 14,25 & 12,11 & 4,17 & 6,89 \\
& GPDS750 & 8,25 & 15,35 & 10,87 & 5,64 & 6,82 \\
\hline
\end{tabular}

Table 2. Results using LBP features.

\begin{tabular}{l|l|r|r|r|r|r}
\hline$\#$ & Dataset & FAR(\%) & FRR(\%) & EER(\%) & $\sigma$-FAR & $\sigma$-FRR \\
\hline 5 & MCYT & 6,72 & 25,79 & 14,34 & 3,92 & 9,20 \\
& GPDS100 & 6,20 & 25,98 & 14,94 & 3,73 & 7,96 \\
& GPDS750 & 6,00 & 27,67 & 15,57 & 4,34 & 8,79 \\
10 & MCYT & 11,51 & 13,71 & 12,06 & 3,22 & 9,76 \\
& GPDS100 & 11,95 & 11,52 & 11,79 & 4,06 & 5,53 \\
& GPDS750 & 8,29 & 14,16 & 10,45 & 4,40 & 6,74 \\
\hline
\end{tabular}

Table 3. Results using a combination of GLCM and LBP features (GLCM+LBP).

\begin{tabular}{l|l|r|r|r|r|r}
\hline$\#$ & Dataset & FAR(\%) & FRR(\%) & EER(\%) & $\sigma$-FAR & $\sigma$-FRR \\
\hline 5 & MCYT & 3,94 & 25,77 & 12,67 & 2,90 & 9,20 \\
& GPDS100 & 6,30 & 18,55 & 11,71 & 3,27 & 7,45 \\
& GPDS750 & 6,69 & 19,63 & 12,41 & 5,50 & 7,87 \\
10 & MCYT & 9,12 & 11,83 & 9,80 & 3,42 & 9,42 \\
& GPDS100 & 11,25 & 6,27 & 9,42 & 3,63 & 4,40 \\
& GPDS750 & 8,51 & 6,79 & 7,88 & 4,31 & 4,73 \\
\hline
\end{tabular}

Table 4. Results using Wavelet decomposition and a combination of GLCM+LBP.

\section{Conclusions}

The use of Wavelet transform as a complement to the features based on texture analysis, has improved the system performance. A single level of decomposition is sufficient to achieve acceptable results. A multilevel Wavelet decomposition significantly increases the computational cost without providing improved results.

Regarding the combination of features, it can be concluded that the joint use of features based on texture analysis improves system performance. The combination made at the level of features, offers better results with respect to use of individual traits. Additionally, this combination of features is enhanced when using the Wavelet transform as a complement to 
it. Using Wavelet transform as a previous step to the characterization of the signature using the combination of features provides the best results presented in this paper in terms of EER values and variability for different databases containing samples made with different type of ink.

In an image containing a handwritten signature, most of the pixels belongs the background. This large percentage of elements not provide information about the signature. Using the blocks analysis it is possible to perform a local analysis, allowing to detect areas where there are no traces of the signature. In this way, characterization is done mainly on the elements belonging to different strokes, improving system performance.

\subsection{Future research}

It is important to conduct a study to verify the results obtained in this study for more complex environments, such as images in which the signature is contaminated with seals, or characters, especially case the checks. It is necessary to consider whether the procedures for the signature segmentation in such images affects gray level information.

As seen, the combination of features can improve system performance. As mentioned, this combination was performed at feature level, in this way, would be highly relevant to study the performance of the system when the combination is done at score level. It is also of interest to study the combination of features based on gray levels with those based on binary information. If takes into account that so far the best results were obtained in works where it is used features of the latter type, combining features of different nature could improve the overall performance of a signature verification system.

\section{References}

Alonso-Fernandez, F., Fairhurst, M. C., Fierrez, J. \& Ortega-Garcia, J. (2007). Automatic measures for predicting performance in off-line signature, IEEE Proceedings International Conference on Image Processing,, Vol. 1, pp. 369-372.

Bertolini, D., Oliveira, L., Justino, E. \& Sabourin, R. (2009). Reducing forgeries in writer-independent off-line signature verification through ensemble of classifiers, Pattern Recognition 43(1): 387-396.

Bhagvati, C. \& Haritha, D. (2005). Classification of Liquid and Viscous Inks using HSV Colour Space, Proceedings of the Eighth International Conference on Document Analysis and Recognition, IEEE Computer Society, Washington, DC, USA, pp. 660-664.

Conners, R. W. \& Harlow, C. A. (1980). A theoretical comparison of texture algorithms, IEEE Transaction on Pattern Analysis and Machine Intelligence 2(3): 204-222.

Ellen, D. (1997). The scientific examination of documents : methods and techniques, 2nd ed. edn, Taylor \& Francis, London ; Bristol, PA :.

Ferrer, M., Travieso, C., J.F.Vargas \& Alonso, J. (2006). Aplicación del Kernel de Fisher para la verificación de firmas manuscritas, Terceras Jornadas de Reconocimiento Biométrico de Personas, pp. 23-36.

Fierrez-Aguilar, J., Alonso-Hermira, N., Moreno-Marquez, G. \& Ortega-Garcia, J. (2004). An off-line signature verification system based on fusion of local and global information, Proceedings of the Workshop on Biometric Authentication, Springer LNCS-3087, pp. 298-306.

Franke, K., Bünnemeyer, O. \& Sy, T. (2002). Ink texture analysis for writer identification, Proceedings of the Eighth International Workshop on Frontiers in Handwriting Recognition, IEEE Computer Society, Washington, DC, USA, p. 268. 
Franke, K. \& Grube, G. (1998). The automatic extraction of pseudodynamic information from static images of handwriting based on marked grayvalue segmentation (extended version), Journal of Forensic Document Examination 11(3): 17-38.

Franke, K. \& Rose, S. (2004). Ink-deposition model: The relation of writing and ink deposition processes, Proceedings of the Ninth International Workshop on Frontiers in Handwriting Recognition, IEEE Computer Society, Washington, DC, USA, pp. 173-178.

Freire, M., Fierrez, J., Martinez-Diaz, M. \& Ortega-Garcia, J. (2007). On the applicability of off-line signatures to the fuzzy vault construction, Proceedings of the Ninth International Conference on Document Analysis and Recognition,, Vol. 2, IEEE Computer Society, pp. 1173-1177.

Gilperez, A., Alonso-Fernandez, F., Pecharroman, S., Fierrez, J. \& Ortega-Garcia, J. (2008). Off-line signature verification using contour features, Proceedings International Conference on Frontiers in Handwriting Recognition.

Güler, I. \& Meghdadi, M. (2008). A different approach to off-line handwritten signature verification using the optimal dynamic time warping algorithm, Digital Signal Processing 18(6): 940-950.

Haralick, R. M. (1979). Statistical and structural approaches to texture, Proceedings of the IEEE 67(5): 786-804.

URL: http://ieeexplore.ieee.org/xpls/abs_all.jsp?arnumber $=1455597$

He, D., Wang, L. \& Guibert, J. (1987). Texture feature extraction, Pattern Recognition Letters 6(4): 269-273.

Liu, S. \& Silverman, M. (2001). A practical guide to biometric security technology, IEEE IT Professional 3(1): 27-32.

Marcel, S., Rodriguez, Y. \& Heusch, G. (2007). On the recent use of local binary patterns for face authentication, International Journal on Image and Video Processing Special Issue on Facial Image Processing .

Nikam, S. \& Agarwal, S. (2008). Texture and wavelet-based spoof fingerprint detection for fingerprint biometric systems, Emerging Trends in Engineering and Technology, 2008. ICETET '08. First International Conference on, pp. 675 -680.

Ojala, T., Pietikäinen, M. \& Mäenpää, T. (2002). Multiresolution gray-scale and rotation invariant texture classification with local binary patterns, IEEE Transactions on Pattern Analysis and Machine Intelligence 24(7): 971-987.

Prakash, H. \& Guru, D. (2009). Relative orientations of geometric centroids for off-line signature verification, International Conference on Advances in Pattern Recognition, 1: 201-204.

T., M. (2003). The local binary pattern approach to texture analysis - extensions and applications., PhD thesis. Dissertation. Acta Univ Oul C 187, 78 p + App.

URL: http://herkules.oulu.fi/isbn9514270762/

Thanasoulias, N., Evmiridis, N. \& Parisis, N. (2003). Multivariate chemometrics for the forensic discrimination of blue ball-point pen inks based on their vis spectra, Forensic science international 138: 75-84.

TowerGroup (Accessed: October, 2008 ). Press releases. URL: http://www.towergroup.com/research/news/news.htm?newsId $=4500$

Trivedi, M., Harlow, C., Conners, R. \& Goh, S. (1984). Object detection based on gray level co-occurrence, Computer Vision, Graphics, and Image Processing 28(3): 199-219.

Vargas, J., Ferrer, M., Travieso, C. \& Alonso, J. (2007). Off-line Handwritten Signature GPDS-960 Corpus, Proceedings of the Ninth International Conference on Document Analysis and Recognition 2007, pp. 764-768. 


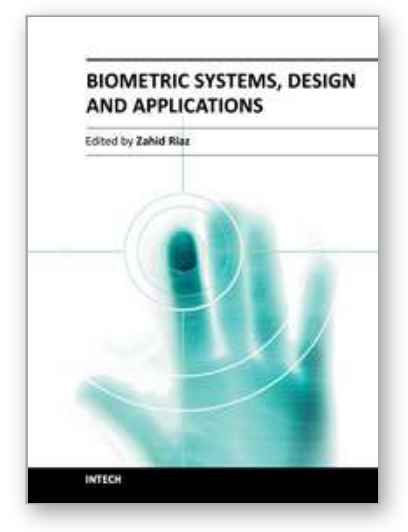

\author{
Biometric Systems, Design and Applications \\ Edited by Mr Zahid Riaz
}

ISBN 978-953-307-542-6

Hard cover, 262 pages

Publisher InTech

Published online 21, October, 2011

Published in print edition October, 2011

Biometric authentication has been widely used for access control and security systems over the past few years. The purpose of this book is to provide the readers with life cycle of different biometric authentication systems from their design and development to qualification and final application. The major systems discussed in this book include fingerprint identification, face recognition, iris segmentation and classification, signature verification and other miscellaneous systems which describe management policies of biometrics, reliability measures, pressure based typing and signature verification, bio-chemical systems and behavioral characteristics. In summary, this book provides the students and the researchers with different approaches to develop biometric authentication systems and at the same time includes state-of-the-art approaches in their design and development. The approaches have been thoroughly tested on standard databases and in real world applications.

\title{
How to reference
}

In order to correctly reference this scholarly work, feel free to copy and paste the following:

Jesus F. Vargas and Miguel E. Ferrer (2011). Texture Analysis for Off-Line Signature Verification, Biometric Systems, Design and Applications, Mr Zahid Riaz (Ed.), ISBN: 978-953-307-542-6, InTech, Available from: http://www.intechopen.com/books/biometric-systems-design-and-applications/texture-analysis-for-off-linesignature-verification

\section{INTECH}

open science | open minds

\author{
InTech Europe \\ University Campus STeP Ri \\ Slavka Krautzeka 83/A \\ 51000 Rijeka, Croatia \\ Phone: +385 (51) 770447 \\ Fax: +385 (51) 686166 \\ www.intechopen.com
}

\author{
InTech China \\ Unit 405, Office Block, Hotel Equatorial Shanghai \\ No.65, Yan An Road (West), Shanghai, 200040, China \\ 中国上海市延安西路65号上海国际贵都大饭店办公楼 405 单元 \\ Phone: +86-21-62489820 \\ Fax: $+86-21-62489821$
}


(C) 2011 The Author(s). Licensee IntechOpen. This is an open access article distributed under the terms of the Creative Commons Attribution 3.0 License, which permits unrestricted use, distribution, and reproduction in any medium, provided the original work is properly cited. 